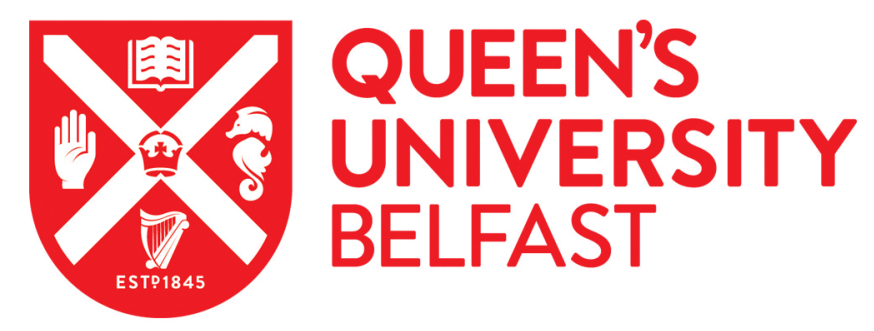

\title{
The effects of transients on photospheric and chromospheric power distributions
}

Samanta, T., Henriques, V. M. J., Banerjee, D., Prasad, S. K., Mathioudakis, M., Jess, D., \& Pant, V. (2016). The effects of transients on photospheric and chromospheric power distributions. The Astrophysical Journal, 828(23). https://doi.org/10.3847/0004-637X/828/1/23

\section{Published in:}

The Astrophysical Journal

Document Version:

Publisher's PDF, also known as Version of record

Queen's University Belfast - Research Portal:

Link to publication record in Queen's University Belfast Research Portal

Publisher rights

(c) 2016. The American Astronomical Society. All rights reserved

\section{General rights}

Copyright for the publications made accessible via the Queen's University Belfast Research Portal is retained by the author(s) and / or other copyright owners and it is a condition of accessing these publications that users recognise and abide by the legal requirements associated with these rights.

Take down policy

The Research Portal is Queen's institutional repository that provides access to Queen's research output. Every effort has been made to ensure that content in the Research Portal does not infringe any person's rights, or applicable UK laws. If you discover content in the Research Portal that you believe breaches copyright or violates any law, please contact openaccess@qub.ac.uk. 


\title{
THE EFFECTS OF TRANSIENTS ON PHOTOSPHERIC AND CHROMOSPHERIC POWER DISTRIBUTIONS
}

\author{
T. Samanta ${ }^{1}$, V. M. J. Henriques ${ }^{2}$, D. Banerjee ${ }^{1,3}$, S. Krishna Prasad ${ }^{2}$, M. Mathioudakis ${ }^{2}$, D. Jess $^{2}$, and V. Pant ${ }^{1}$ \\ ${ }^{1}$ Indian Institute of Astrophysics, Koramangala, Bangalore 560034, India; tsamanta@iiap.res.in \\ ${ }^{2}$ Astrophysics Research Centre, School of Mathematics and Physics, Queen’s University Belfast, Belfast BT7 1NN, UK; v.henriques@qub.ac.uk \\ ${ }^{3}$ Center of Excellence in Space Sciences, IISER Kolkata, India \\ Received 2015 September 14; revised 2016 April 20; accepted 2016 April 20; published 2016 August 24
}

\begin{abstract}
We have observed a quiet-Sun region with the Swedish $1 \mathrm{~m}$ Solar Telescope equipped with the CRISP Imaging SpectroPolarimeter. High-resolution, high-cadence, $\mathrm{H} \alpha$ line scanning images were taken to observe different layers of the solar atmosphere from the photosphere to upper chromosphere. We study the distribution of power in different period bands at different heights. Power maps of the upper photosphere and the lower chromosphere show suppressed power surrounding the magnetic-network elements, known as "magnetic shadows." These also show enhanced power close to the photosphere, traditionally referred to as "power halos." The interaction between acoustic waves and inclined magnetic fields is generally believed to be responsible for these two effects. In this study we explore whether small-scale transients can influence the distribution of power at different heights. We show that the presence of transients, like mottles, Rapid Blueshifted Excursions (RBEs), and Rapid Redshifted Excursions (RREs), can strongly influence the power maps. The short and finite lifetime of these events strongly affects all power maps, potentially influencing the observed power distribution. We show that Doppler-shifted transients like RBEs and RREs that occur ubiquitously can have a dominant effect on the formation of the power halos in the quiet Sun. For magnetic shadows, transients like mottles do not seem to have a significant effect on the power suppression around 3 minutes, and wave interaction may play a key role here. Our high-cadence observations reveal that flows, waves, and shocks manifest in the presence of magnetic fields to form a nonlinear magnetohydrodynamic system.
\end{abstract}

Key words: Sun: corona - Sun: oscillations - Sun: transition region - Sun: UV radiation

Supporting material: animations

\section{INTRODUCTION}

The solar chromosphere is a layer above the visible solar surface spanning over approximately a thousand kilometers in height. It plays an important role in understanding the interaction between the relatively cool photospheric plasma and the hot multi-million degree corona. Small-scale magnetic flux concentrations at the boundaries of supergranular cells extend upwards into the chromosphere. These flux tubes expand into funnel-like structures with height due to a decrease in the ambient gas pressure. Some field lines locally connect within the photosphere and produce a canopy-like structure in the chromosphere and some of them reach the corona. The chromosphere is still a poorly understood layer where flows, waves, and shocks manifest in the presence of magnetic fields to form an often nonlinear magnetohydrodynamic system.

Waves in the solar atmosphere are studied with great interest as they carry mechanical energy and also provide insight into the physical parameters through seismology (Roberts 2000; Banerjee et al. 2007; Zaqarashvili \& Erdélyi 2009; De Moortel \& Nakariakov 2012; Jess et al. 2015). Oscillations are observed ubiquitously throughout the solar atmosphere and are often interpreted in terms of various magnetohydrodynamic (MHD) modes. Acoustic waves (p-modes), which are generated inside the Sun, are generally trapped inside it. These waves can freely propagate from the surface into the atmosphere if they have periods shorter than 3.2 minutes $(5.2 \mathrm{mHz})$, which is known as the acoustic cut-off period. The longer periods generally do not propagate to greater heights and are, instead, evanescent. There is substantial observational evidence for the presence of long period oscillations (Vecchio et al. 2007; Kontogiannis et al. 2010a, 2014; Bostanc1 et al. 2014) in the chromosphere around network magnetic elements. It appears that the presence of a strong network magnetic field changes the scenario (Rosenthal et al. 2002; Bogdan et al. 2003). Roberts (1983), Centeno et al. (2006), and Khomenko et al. (2008) argue that strong magnetic fields change the radiative relaxation time, which can increase the cut-off period significantly. There are also suggestions that the field inclination plays a very important role in long-period wave propagation (Carlsson \& Bogdan 2006; Jess et al. 2013; Kontogiannis et al. 2014). Heggland et al. (2011) show that for long-period wave propagation, the field inclination is much more important than the radiative relaxation time effect. Highly inclined magnetic fields significantly increase the cut-off period and create magnetoacoustic portals (Jefferies et al. 2006) for the propagation of long-period waves in the chromosphere. This is commonly referred to as leakage of photospheric oscillations into the chromosphere (De Pontieu et al. 2004). The "leakage" of long-period photospheric oscillations takes place through magnetic network elements through restricted areas. Recent studies show that a good fraction of power is present above the cut-off period at higher layers around the quiet magnetic network elements (Judge et al. 2001; McIntosh et al. 2003; Moretti et al. 2007; Vecchio et al. 2007; Kontogiannis et al. 2010b). Two-dimensional power maps of period bands around 3 minutes reveal two distinct phenomena above network and around elements. One is known as "power halos," which are upper-photospheric regions where the wave power is enhanced. The other is "magnetic shadows," which refer to the regions of the power suppression around network elements in the chromosphere.

Many researchers have suggested that the interaction between the acoustic waves and the magnetic fields is 




Figure 1. Images of a quiet region as seen in different layers of the solar atmosphere along with the corresponding magnetogram from photosphere at the bottom. Bottom to top: line-of-sight (LOS) magnetogram obtained by using Fe $6302 \AA$ Stokes V profiles, visible continuum, and narrow-band filter images taken at different positions across the $\mathrm{H} \alpha$ line profile as indicated $(\mathrm{H} \alpha+0.906 \AA, \mathrm{H} \alpha+0.543 \AA, \mathrm{H} \alpha+0.362 \AA$ and $\mathrm{H} \alpha$ core). The long tick marks on the magnetogram represent $10 \mathrm{Mm}$ intervals. The region outlined by the dotted line covers a network region is further studied in Figures 5,6 and 11 .

(An animation of this figure is available.)

responsible for the formation of magnetic shadows and power halos (Judge et al. 2001; McIntosh et al. 2003; Moretti et al. 2007; Vecchio et al. 2007; Kontogiannis et al. 2010b). It was proposed that the upward propagating acoustic waves change their nature at the magnetic canopy, a layer where the gas pressure becomes equal to the magnetic pressure, and undergo mode conversion and transmission processes (Nutto et al. 2010). Simulations show that acoustic waves generally transfer their energy partly to the slow magnetoacoustic waves (mode transmission) and partly to the fast magnetoacoustic waves (mode conversion) at the canopy (Nutto et al. 2012b). Due to high velocity gradients, the fast mode undergoes reflection at the canopy and increases the oscillation power at lower heights, creating power halos (Nutto et al. 2012a). In contrast, the slow mode continues to propagate along the slanted magnetic field lines. Kontogiannis et al. (2014) argue that the key parameter in mode conversion mechanism is the attack angle (the angle between the direction of wave propagation and the magnetic field) and the period of the acoustic waves. They show that the transmission is generally favored at small attack angles and long periods, while the conversion dominates when the period is small and the attack angle is large, which causes the power halos and magnetic shadows to form around magnetic network regions.

However, most of the earlier studies that put forward the theory based on magnetoacoustic-wave reflection did not consider the effect of transients nor the evolution of magnetic fields and other factors leading to changes in the visible chromospheric canopy. Earlier observations were also limited by temporal resolution that were too low to study the influence of short-lived transients in detail. With our high spatial and temporal resolution observations taken with the $1 \mathrm{~m}$ Swedish Solar Telescope (SST), we revisit the subject and attempt to provide an alternative interpretation for the formation of the magnetic shadow and power halo. Section 2 describes the observations along with data reduction procedures. Section 3 provides results in terms of power distribution at several heights in the chromosphere. Section 4 deals with possible scenarios which can explain our observations and compares with earlier interpretations. Finally, conclusions are drawn in Section 5.

\section{OBSERVATIONS AND DATA PROCESSING}

Observations of a quiet-Sun region were made on 2013 May 3, from 09:06 UT to 09:35 UT using the CRisp Imaging SpectroPolarimeter (CRISP; Scharmer 2006; Scharmer et al. 2008) at the SST (Scharmer et al. 2003a). Images were taken at seven wavelength positions scanning through the $\mathrm{H} \alpha$ line, at $-0.906,-0.543,-0.362,0.000,0.362,0.543$, and $+0.906 \AA$ from the line core, corresponding to a velocity range of -41 to $+41 \mathrm{~km} \mathrm{~s}^{-1}$. Adaptive optics was employed in the observations with the upgraded 85 electrode system (Scharmer et al. 2003b).

All the data were reconstructed using Multi-Object MultiFrame Blind Deconvolution (MOMFBD; Löfdahl 2002; van Noort et al. 2005), with the 51 Karhunen-Loève modes sorted by order of atmospheric significance and $88 \times 88$ pixel subfields. An early version of the pipeline described in de la Cruz Rodríguez et al. (2015) was used. Destretching (Shine et al. 1994) was used together with auxiliary wide-band objects for consistent co-alignment of different narrow-band passbands, as described in Henriques (2012). Spatial sampling is 0 . 058 pixel $^{-1}$, and the spatial resolution reaches up to 0 ". 16 in $\mathrm{H} \alpha$ covering a field of view (FOV) of $40 \times 40 \mathrm{Mm}^{2}$. After reconstruction, the cadence of a full spectral scan was $1.34 \mathrm{~s}$. In this work, we also made use of wide-band images obtained with the CRISP reference camera. This camera is behind the $\mathrm{H} \alpha$ pre-filter but before the double Fabry-Pérot. The pre-filter has a $1 \mathrm{~nm}$ passband centered at the core of the line. The images from this camera provide the anchor channel for MOMFBD reconstruction and the reference for all postreconstruction destretch-based techniques. The vast majority of the light contributing to the images from this camera come from the photospheric wings of the $\mathrm{H} \alpha$ line. The cadence of the wide band was also $1.34 \mathrm{~s}$.

Line-of-Sight (LOS) magnetograms were produced from the Stokes V output of Fe $6301 \mathrm{~A}$ spectral scans (taken at 16 

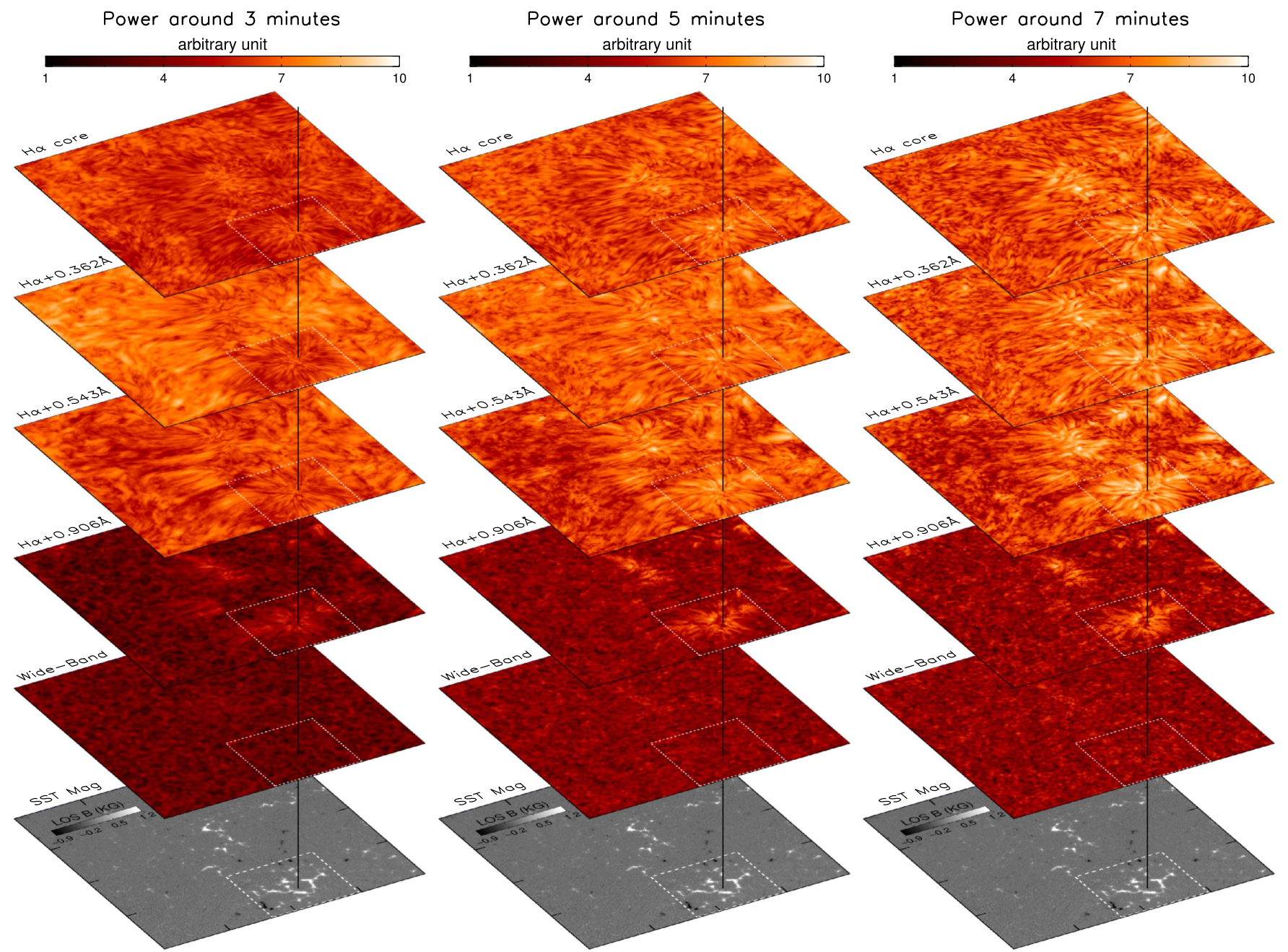

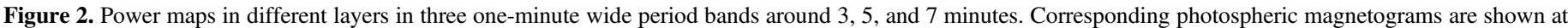
the bottom. The long tick marks on the magnetogram represent $10 \mathrm{Mm}$ intervals.

wavelength positions) using the center of gravity (COG) method (Rees \& Semel 1979; Uitenbroek 2003). These scans were acquired at a cadence of $\sim 5$ minutes over the same FOV. The same $\mathrm{H} \alpha$ camera was used for obtaining Stokes $\mathrm{V}$ data. Hence there were gaps of $\sim 27 \mathrm{~s}$ at $\sim 5,11,16$, and 21 minutes of observation. We have interpolated these data gaps using a spline function to obtain a regular cadence for time series analysis. Note that the treatment via spline-fitting is smoothly "bridging" intensity in the time series whereas Rapid Blueshifted Excursion (RBE)/Rapid Redshifted Excursions (RREs) and mottles cause strong dips in the intensity. Further details on the observations and data reduction are given in Kuridze et al. (2015) and Henriques et al. (2016).

$\mathrm{H} \alpha$ core maps were produced using Doppler compensation. For this, at first, we increased the line profile sampling by a factor of 10 times more than the original using spline interpolation, then the minimum value of the profile is calculated at each pixel to produce the Doppler-compensated $\mathrm{H} \alpha$ core maps. This procedure minimizes the effects of strong flows that might shift the position of the line core, and thus best represents the emission coming from the line-forming region (see, e.g., Jess et al. 2010). The LOS Doppler velocity maps were determined by the COG method.
The $\mathrm{H} \alpha$ line core forms at the chromosphere and the wings form at lower atmospheric heights (Leenaarts et al. 2006, 2012). Filtergram images taken at different positions of the $\mathrm{H} \alpha$ line sample, on average, different atmospheric layers and are shown in Figure 1. A time lapse movie of this figure is also available. The movie clearly shows the presence of transients.

\section{RESULTS}

\subsection{Spatially Resolved Power Distributions in Different Period Bands}

We investigate the oscillation properties of the different layers by constructing power maps. The construction of the power maps was preceded by the removal of a background trend from each light curve to obtain the relative percentage intensity variations $\left(I_{R}\right)$ given by $I_{R}=\left(I-I_{\mathrm{bg}}\right) * I_{\mathrm{bg}}{ }^{-1} * 100$, where $I$ is the original intensity and $I_{\mathrm{bg}}$ is the background trend. The background trend, $I_{\mathrm{bg}}$, is computed from the original light curve over a $600 \mathrm{~s}$ running average, which when subtracted from the original time series allows intensity fluctuations shorter than 10 minutes to be more readily identified. The resultant light curves are then subjected to wavelet analysis (Torrence \& Compo 1998) and the global wavelet power 


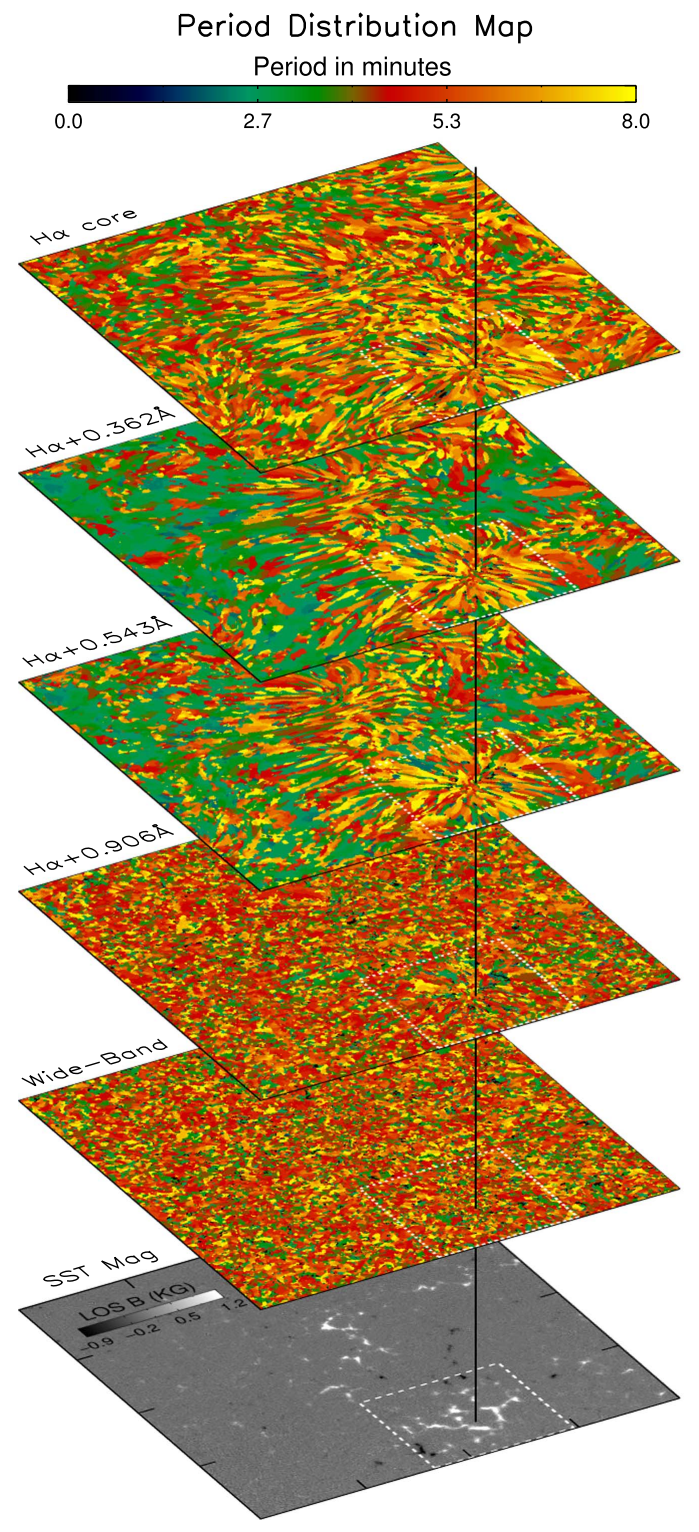

Figure 3. Distribution of dominant periods in different layers along with the corresponding magnetogram at the bottom. The green, red, and yellow colors roughly represent periods around 3,5 , and 7 minutes, respectively.

spectrum is calculated at each pixel. An example of the computed relative intensity variations and the corresponding wavelet analysis results at a single pixel is shown in Figure 5. Power maps were constructed for 3,5 , and 7 minute periods from the global wavelet power spectrum by averaging the power in one-minute bands around each period. Figure 2 displays these maps stacked in ascending order of atmospheric height for each band. A co-spatial photospheric magnetogram is also shown at the bottom panel for comparison. Figure 2 reveals that power is suppressed in rosettes over the network in the 3 minute band at the lower chromosphere $(\mathrm{H} \alpha+0.543$ and $\mathrm{H} \alpha+0.362 \AA$ ) and enhanced close to the photosphere $(\mathrm{H} \alpha+0.906 \AA)$ in all bands. These phenomena are known as magnetic shadows (Judge et al. 2001; McIntosh et al. 2003; Moretti et al. 2007; Vecchio et al. 2007; Kontogiannis et al. 2010b, 2014) and power halos (Kontogiannis et al. 2010a), respectively.
We also make period maps to study the spatial distribution of dominant periods in each layer. The period at maximum power above the $99 \%$ significance level is taken as the dominant period at each pixel to construct these maps. The significance levels are calculated assuming white noise (Torrence \& Compo 1998). The period distribution maps are shown in Figure 3 along with the photospheric magnetogram. As in Figures 2 and 3, only the maps produced from the red wings of $\mathrm{H} \alpha$ are displayed since the blue-wing maps look very similar. It is evident from the figure that in the layers dominated by the photosphere (wide-band and $\mathrm{H} \alpha+0.906 \AA$ ), the well-known 5-minute photospheric p-mode oscillation is dominant. At larger heights $(\mathrm{H} \alpha+0.543$ and $\mathrm{H} \alpha+0.362 \AA)$, the 3-minute period becomes dominant for most of the FOV, with the exception of the neighborhood of the network magnetic element where the longer (5-7 minutes) periods become dominant. The distribution of periods (see Figure 4(E)) in the $\mathrm{H} \alpha$ Doppler velocity maps show that the 3-minute oscillations cover a wider extent than that in the corresponding period maps computed from the $\mathrm{H} \alpha$-core intensity (see Figure 3). This behavior was observed earlier by De Pontieu et al. (2007). The velocity power maps at different period bands are also shown in Figures 4(B)-(D). It shows enhanced power in the higher period bands around the network and suppressed power at a lower period band ( 3 minutes) at the same region. Power/ period maps were also generated using fast Fourier transform techniques. No significant differences were found when compared to our wavelet results, and hence to avoid duplication we do not include these figures here.

\subsection{Space-Time Plots and Wavelet Analysis}

We have generated spacetime plots to study if the compressible periodic disturbances are propagating along the elongated structures in the network region. Artificial slits are placed radially outward from the center of the rosette structure as shown by a green solid line over the $\mathrm{H} \alpha+0.906 \AA$ image in Figure 5(A). The corresponding spacetime plot is displayed in Figure 5(B) and shows a few alternating dark ridges at the top. The propagation speeds calculated from the slope of one of the ridges is around $120 \mathrm{~km} \mathrm{~s}^{-1}$. These ridges correspond to transient events like RBEs and RREs which are on-disk absorption features generally seen in the red and blue wings of chromospheric lines (Langangen et al. 2008; Rouppe van der Voort et al. 2009). Using the same data set, RBEs and RREs from this region have already been studied by Kuridze et al. (2015). These events have the appearance of high speed jets or blobs and are generally directed outward from a magnetic network bright point with speeds of $50-150 \mathrm{~km} \mathrm{~s}^{-1}$. They can be heated up to transition region (or even coronal) temperatures with a lifetime of 10-120 s and are believed to be the on-disk counterparts of Type II spicules (Pereira et al. 2014; Kuridze et al. 2015; Rouppe van der Voort et al. 2015; Henriques et al. 2016). We select many locations around the network concentrations and find clear signatures of RBEs and RREs repeatedly appearing around the same place. Within $\sim 28$ minutes of our observations they occur 1-15 times (intensity decreases $\gtrsim 1 \sigma$ ) at the same location, with an average of 3-5 times. A closer inspection of the movie shows the clear presence of such transients.

The results of the wavelet analysis for the light curve from the row marked by a dashed line in Figure 5(B), corresponding to position P1 marked in panel (A), are shown in panels (C) to 

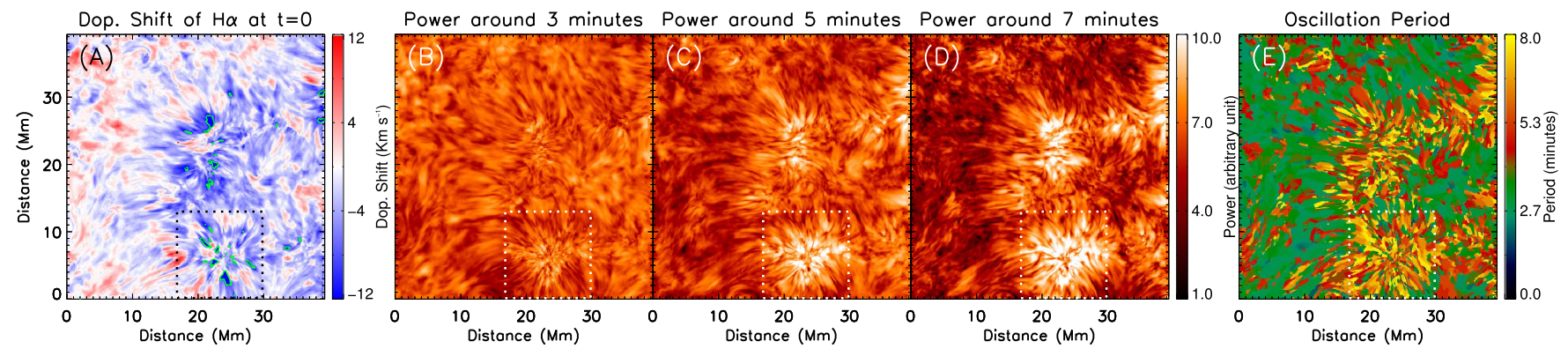

Figure 4. (A): $\mathrm{H} \alpha$ Doppler shift map (a $t=0$ ) obtained using the Center-of-Gravity (COG) method. The display scale is saturated to $\pm 12 \mathrm{~km} \mathrm{~s}^{-1}$ for better view. The value inside the green contours correspond to higher than $\pm 12 \mathrm{~km} \mathrm{~s}^{-1}$. The power maps at different period bands are shown in panels (B)-(D). (E): Distribution of the dominant periods.
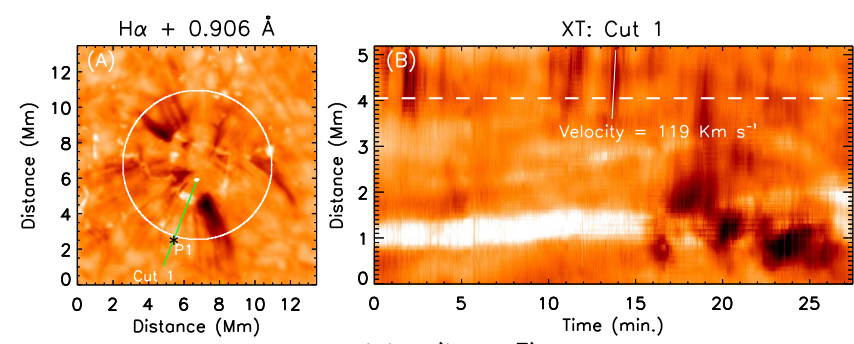

Intensity vs Time

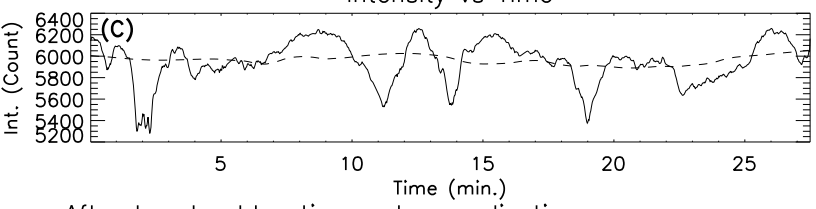

After trend subtraction and normalization

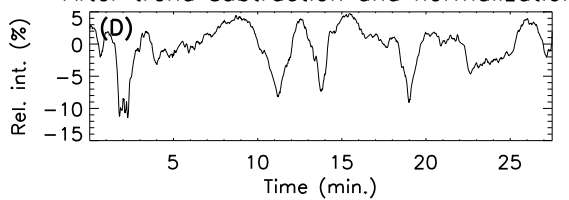

Global Period at max. power (< $10.0 \mathrm{~min}$.) Second highest Peak $\mathrm{P} 2=4.6 \mathrm{~min}$.

Wavelet Power Spectrum

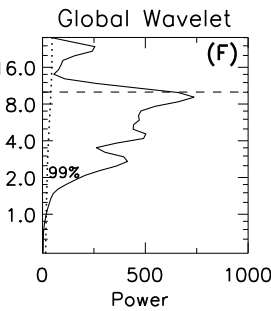

Figure 5. (A): $\mathrm{H} \alpha+0.906 \AA$ image. A cut along the green line is taken to produce the spacetime map shown in $(\mathrm{B})$. The white dot represents the starting point. (B): Temporal evolution along the green line shown in (A). The dashed line corresponds to the position P1 marked by an asterisk in (A). The slanted solid line indicates the track used for measuring the propagation speed. (C): The intensity variation along the dashed line in (B). The overplotted dashed line represents the background trend. (D): Relative intensity variation after trend subtraction and normalization. (E): The wavelet power spectrum of the normalized time series. The overplotted cross-hatched region is the Cone-OfInfluence (COI) with darker color representing higher power. (F): Global wavelet power spectrum. The maximum measurable period, 10 minutes (due to COI), is shown by a horizontal dashed line. The dotted curve shows the $99 \%$ significance level. The two most significant periods identified from the global wavelet power spectrum are printed on top of the global wavelet plot. An animation of panel (A) for a bigger field-of-view and also for $\mathrm{H} \alpha$ blue wing (H $\alpha-0.906 \AA)$ is available.

(Animations ( $\mathrm{a}, \mathrm{b}$ and $\mathrm{c}$ ) of this figure are available.)

(F) of Figure 5. Panel (C) displays the original light curve (solid line) and the background trend (dashed line), while panel (D) displays the relative intensity as defined in Section 3.1. Panels (E) and (F) display the wavelet and global wavelet
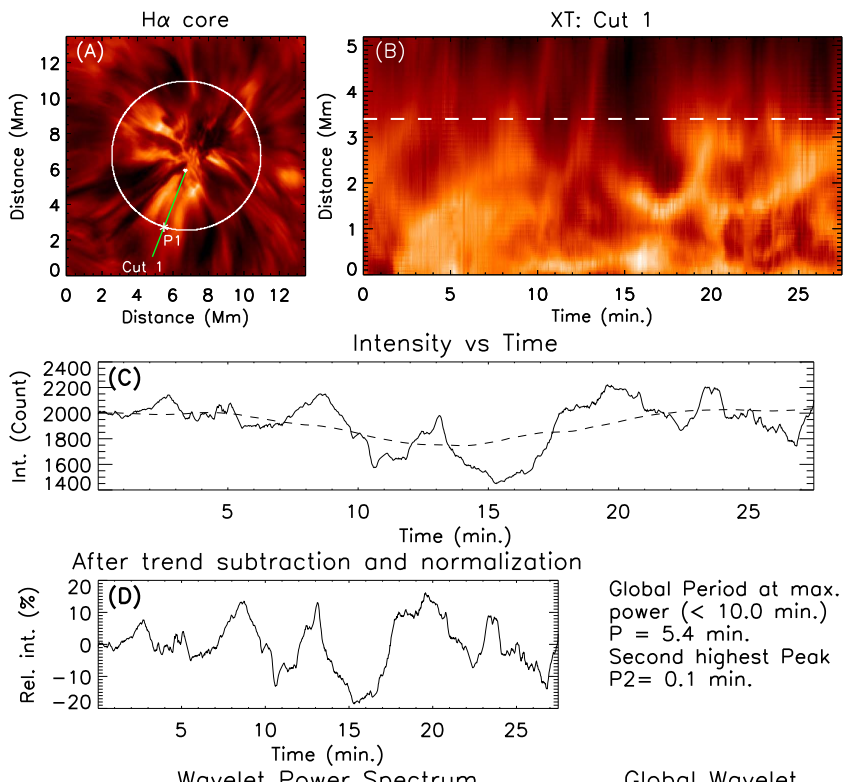

Global Period at max. power $(<10.0 \mathrm{~min}$.) $\mathrm{P}=5.4 \mathrm{~min}$ Second highest Peok $\mathrm{P} 2=0.1 \mathrm{~min}$

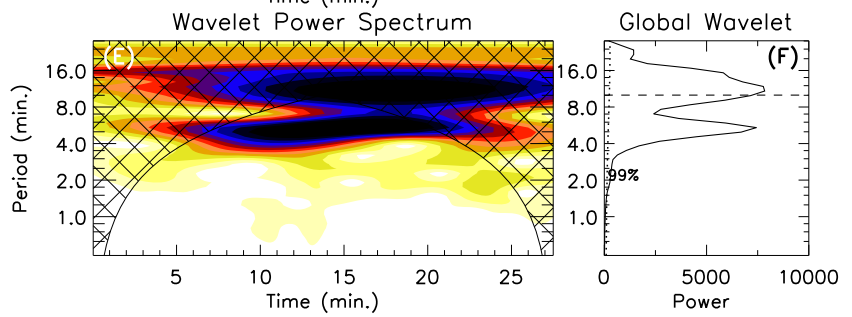

Figure 6. (A) H $\alpha$ core image. Other panels are similar to those in Figure 5.

power spectra. The cross-hatched region in the wavelet plot corresponds to the Cone Of Influence (COI) where the periods identified are not reliable due to the finite length of the time series. The dotted line in the global wavelet plot corresponds to the $99 \%$ significance level assuming a white noise (Torrence \& Compo 1998). The top two periods identified are also listed in the figure. Peaks are found at 9, 4.5, and 2.5 minutes in the global wavelet power. Similar analysis performed over this region in the $\mathrm{H} \alpha$ core shows a peak in power at 5.4 minutes (Figure 6). Figures 5 and 6 indicate the presence of quasiperiodic fluctuations in intensity. The fluctuations in the $\mathrm{H} \alpha$ core are probably caused by the longer lifetime of mottles (3-15 minutes, Tsiropoula et al. 2012). We emphasize that we placed several slits in this region (both in the $\mathrm{H} \alpha$ core and $\mathrm{H} \alpha+0.362 \AA$ scan positions), and our analysis detects oscillation periods around 3-9 minutes. However, the nature of the ridges is not, generally, periodic but rather quasi- 


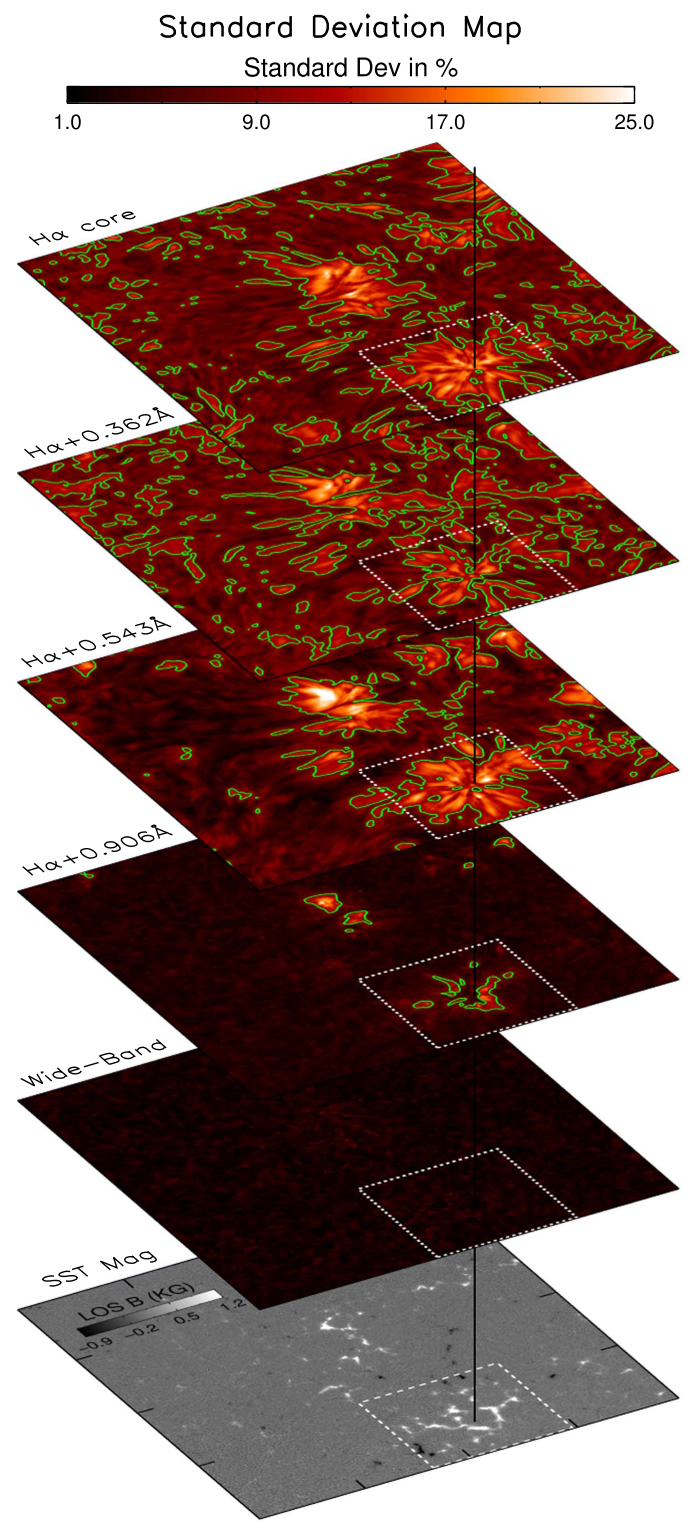

Figure 7. Standard deviation maps in different layers constructed from the normalized percentage standard deviation of the intensity time series at each pixel. The corresponding magnetogram is also shown at the bottom. The green contours enclose regions with a standard deviation of $10 \%$ or more.

periodic. The dark ridges generally show high intensity drops compared to the background, which could be attributed to the outward motion of the mottles.

\subsection{Standard Deviation}

We also measure the standard deviation of the intensity at each pixel and construct normalized percentage standard deviation maps. The normalized percentage standard deviation $(S)$ is estimated at each pixel by following $S=I_{\text {std }} * I_{\text {avg }}{ }^{-1} * 100$, where $I_{\text {std }}$ and $I_{\text {avg }}$ are the standard deviation and average intensity, respectively. The constructed maps are shown in Figure 7 for different layers. It is clear that close to network regions where we observe transients like dark mottles and RBEs, the normalized percentage standard deviation is quite high. A continuous periodic oscillation of $10 \%$ amplitude (with any period) gives a standard deviation of
$7 \%$. In the figure, the green contours outline the regions with a normalized percentage standard deviation of $10 \%$ or more.

\subsection{Artificially Generated Time Series and Wavelet Analysis}

In this subsection we explore the signatures that will be produced in the power spectrum of a bursty signal. Suppose the observed variations in intensity are due to transient phenomena like RREs and mottles. We find that RREs lower the intensity by $5 \%-30 \%$ below the background and have a lifetime of 10 to $120 \mathrm{~s}$. Chromospheric mottles live for 3-15 minutes (Tsiropoula et al. 2012) and cause a decrease in intensity of $10 \%$ $50 \%$. Here, we have generated artificial time series to investigate the affect of non-periodic signals superimposed upon background oscillatory phenomena (e.g., as captured in our observation). Our main motivation is to compare the oscillation power between the network regions (where RBEs, RREs, and mottles are present) and the internetwork regions. We have considered two different cases: the first case models the effects of transients on the power of photospheric wavelength channels, whereas the second case models the impact of transients on the power obtained from chromospheric channels.

Case-1 (photospheric channels): first, we have generated an artificial time series using a sinusoidal signal with a 5-minute period, which we found to be the dominant period in the photosphere (it is also well known). We found that the average normalized percentage standard deviation in the photospheric internetwork regions is $\sim 2.25 \%$ (see Figure 7 ). In order to match with the observed normalized percentage standard deviation, we have selected the amplitude of the sinusoidal periodic signal to be $3.2 \%$ with respect to a constant background (ignoring all the noise and other high- and lowfrequency fluctuations). We then performed wavelet analysis on this artificial signal to compute the global wavelet power spectrum as a reference, which is shown in the top panel in Figure 8 . This was followed by introducing random fluctuations in the same 5-minute periodic signal. The repetition and the amplitudes of the random fluctuations were selected such that they can mimic the observed light curves (an example of the observed light curve is shown in Figure 5). We find that many of the RREs show intensity drops between $10 \%$ and $25 \%$ compared to the background intensity, whereas some of the weak and strong RREs have intensity drops of less than 5\% and more than $30 \%$, respectively. They generally occur repeatedly at the same location (close to the network) with an average of 3-5 times in 28 minutes. Keeping in mind the observed distribution of the transients (RREs), we have produced light curves while introducing sudden fluctuations (Gaussian-shaped dips) with random repetitions (1-5 times) in the same 5-minute periodic signal. We generated 45 light curves while changing the amplitudes $(10 \%, 20 \%$, and 30\%) and temporal width (FWHM of 20, 40, 60, and $80 \mathrm{~s}$ ) of the Gaussian-shaped dips. We then subjected these modified light curves to wavelet analysis for computing the power spectra. Some representative examples (only for the Gaussian dips of intensity amplitudes drops of $20 \%$ with 1,3 , and 4 time repetitions and FWHM of 40,60 , and $80 \mathrm{~s}$ ) are shown in the Figure 8. We compare the power of the 5-minute oscillation of the reference periodic signal (P_5m in red) with the power of the same signal with fluctuations (P_5m in black). Our analysis shows that the power of the 5-minute period is enhanced 1.1-6.8 times due to the presence of RBE-like random fluctuations in the intensity 


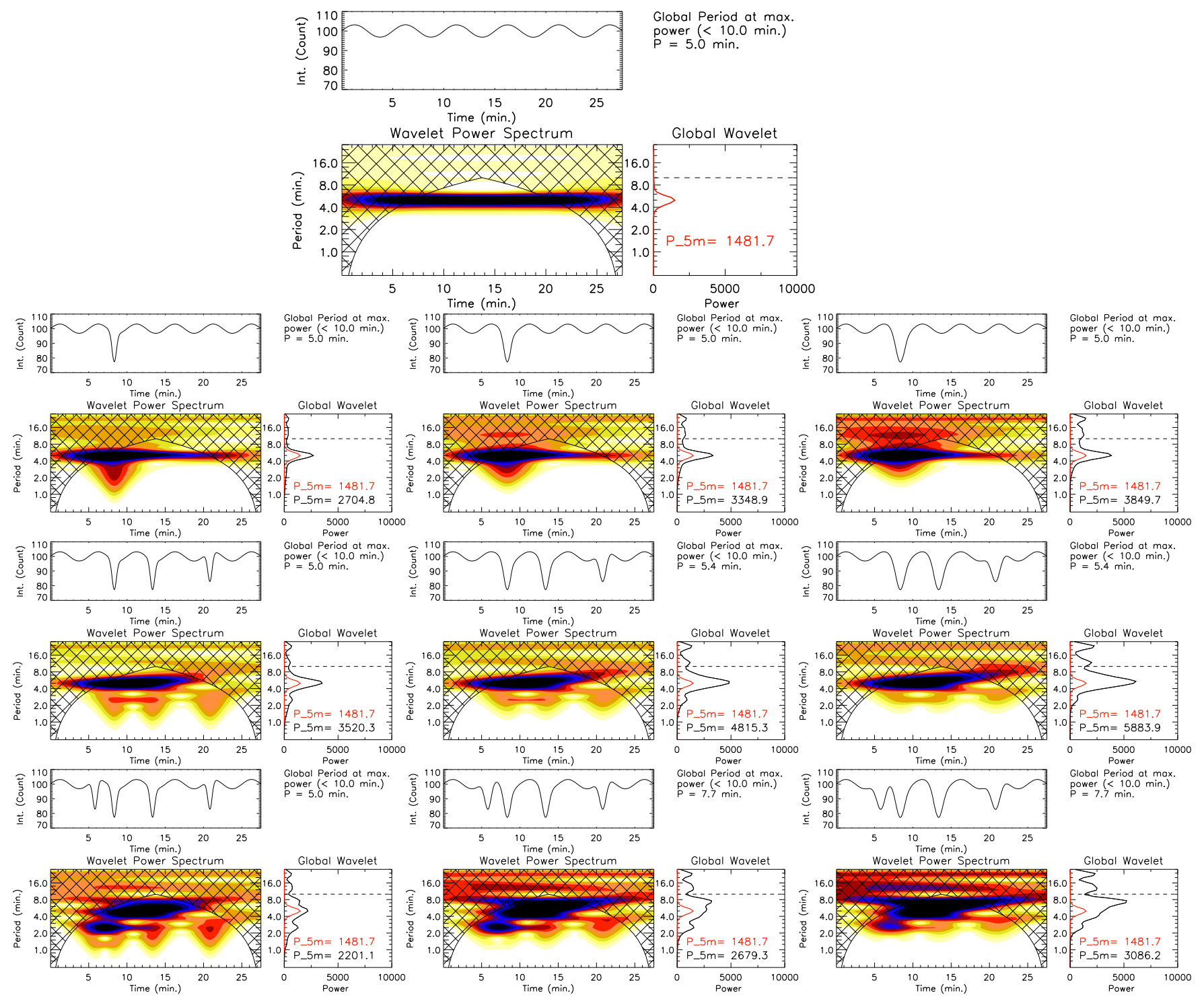

Figure 8. Results of wavelet analysis for the artificially generated light curves. Description of different panels is similar to that in Figure 5. Top panel: wavelet analysis results for a light curve with a periodic sinusoidal signal of 5 minutes. Other panels: wavelet analysis results for several light curves artificially generated by convolving Gaussian-shaped dips in intensity with the periodic signal shown in the top panel. The convolved dips are randomly separated in time with repetition times of 1,3, and 4 across different rows. The FWHM of the dips has been kept at 40,60, and $80 \mathrm{~s}$ across the three columns. The amplitudes of the sinusoidal wave and the Gaussian dips are kept at $3.2 \%$ and $20 \%$, respectively, to a constant background.

and lifetime. The enhancement in power is dependent on the amplitude, temporal width, repetition, and also on the temporal location (phase) of the Gaussian dips. We should point out that we have compared the observed 3-minute power between the regions of enhanced power (network) and internetwork regions in $\mathrm{H} \alpha+0.906 \AA$ and find that the enhancements in power is around 2-5 times compared to the internetwork regions. Additionally, we find that the power in the period band of 2-9 minutes is enhanced which is similar to the observed power distribution.

Case-2 (chromospheric channels): similarly to the first case, we have generated an artificial time series with a periodic sinusoidal signal of period 3 minutes. Here we selected the period of the oscillation to be 3 minutes as the chromospheric internetwork regions $(\mathrm{H} \alpha+0.362 \AA)$ are dominated by a 3minute period. As before, to compare with the observations, we have selected the amplitude of the sinusoid to be $10 \%$ (we find the average normalized percentage standard deviation is $~ 7.4 \%$ in the internetwork regions of the $\mathrm{H} \alpha+0.362 \AA$ layer) with respect to the background. We find that the intensity drops in $\mathrm{H} \alpha+0.362 \AA$ due to the presence of mottles is around $10 \%$ $50 \%$. We have produced 27 light curves while introducing random fluctuations (1-3 Gaussian dips distributed along the whole time series) by changing the amplitudes $(20 \%, 30 \%$, and $40 \%$ ) and temporal width (FWHM of 3, 5, and 7 minutes) followed by wavelet analysis to compute the power. A few examples (for the Gaussian dips with amplitude of $30 \%$ and $40 \%$ only) are shown in Figure 9. We compare the power of the 3-minute oscillation of the pure periodic signal ( $\mathrm{P}_{-} 3 \mathrm{~m}$ in red) with the power of the same signal with fluctuations $\left(\mathrm{P}_{-} 3 \mathrm{~m}\right.$ in black). Our analysis shows that the power of the 3-minute period gets suppressed 2\%-6\% (though the observed magnetic shadow region show around a $60 \%-70 \%$ decrease in the power of the 3-minute oscillation compared to internetwork regions) 


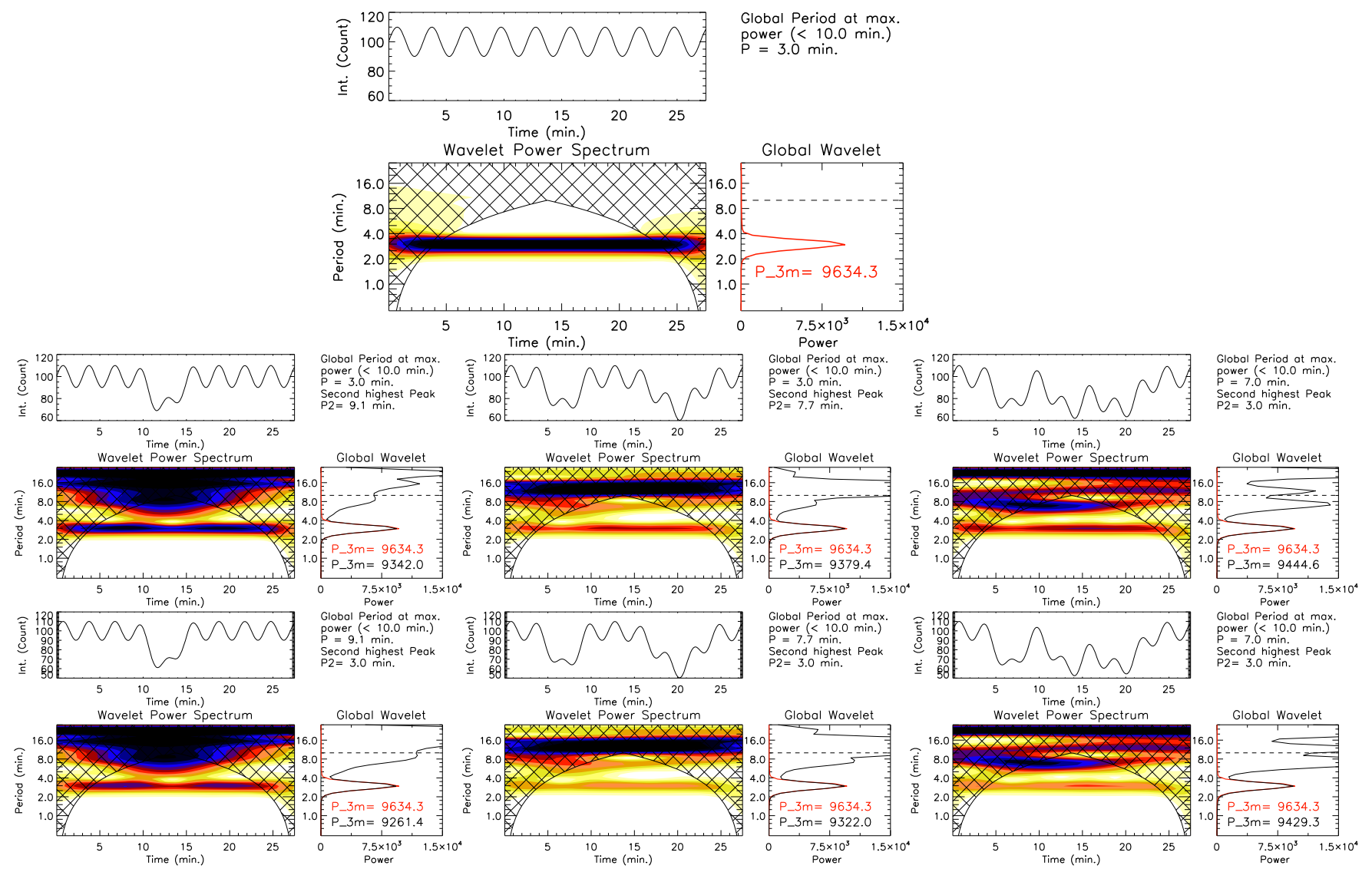

Figure 9. Results of the wavelet analysis for the artificially generated ligthcurves. Description of different panels is similar to that in Figure 5. Top panel: wavelet analysis results for a light curve with a periodic sinusoidal signal of period 3 minutes. Middle panels: wavelet analysis results for several artificially generated light curves made by convolving Gaussian-shaped dips of FWHM 3 minutes; 3 and 5 minutes; and 3, 5, and 7 minutes with the periodic signal shown in the top panel. The amplitudes of the sinusoidal signal and the Gaussian-shaped dips are kept at 10\% and 30\% with respect to the background, respectively. Bottom panels: same as the middle panels but for a $40 \%$ amplitude of the Gaussian-shaped dips with respect to the background.
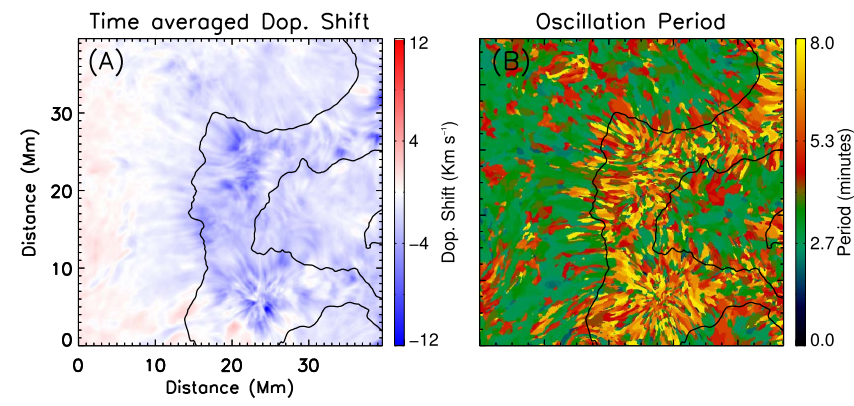

Figure 10. (A): Time-averaged Doppler velocity map of $\mathrm{H} \alpha$ line. (B): Distribution of dominant periods in Doppler velocity oscillations. Contours on both plots represent a dominant period level of 4.5 minutes. The contours are calculated after smoothing the image in panel (B).

due to the presence of random fluctuations like mottles. We also noticed that the power in the period band of 5-9 minutes is generally enhanced due to this kind of sudden fluctuation. Hence, a sudden drop in intensity with a random distribution in time can lead to significant power at different periods. One important thing to note here is that the periods mainly depend on the distribution of the intensity drops and they are generally longer than their FWHM. We should point out that sometimes the power gets enhanced depending on the phase of the Gaussian dips with respect to the continuous 3-minute periodic sinusoid.

\subsection{Time-averaged Doppler Shift and Material Outflows}

The time-averaged Doppler velocity provides very important information on the statistical properties of the dynamics. Figure 10(A) shows the time-averaged Doppler velocity map of the whole FOV. The overplotted contours outline a region with a dominant periodicity of 4.5 minutes as shown in the perioddistribution map (Figure 10(B)). It can be seen that within this region, above the network, the average Doppler velocity is blueshifted $\left(\sim 5 \mathrm{~km} \mathrm{~s}^{-1}\right)$.

The evolution of a portion of the network region is shown in Figure 11. The white box marked in the left panel is our region of interest for temporal variations. The upper panels display the intensity and the bottom panels display the Doppler velocity as captured in one-minute intervals. This figure shows that when dark mottles first start appearing, they are blueshifted but with time the mottles evolve and become bright and redshifted. It is possible that mottles are nothing but strong material outflows like Type I spicules. They appear similar to Type I spicular flows following parabolic paths. The material moves outward, causing a blueshift which turns to redshift when the material falls back on the solar surface.

\section{DISCUSSION}

As pointed out in the introduction, the interaction between acoustic waves and the magnetic field are responsible for the formation of magnetic shadows and power halos (Judge et al. 


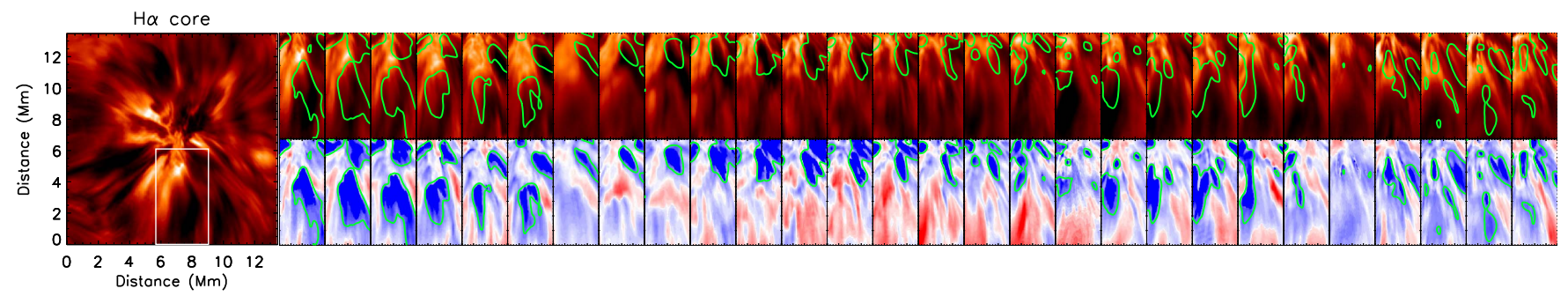

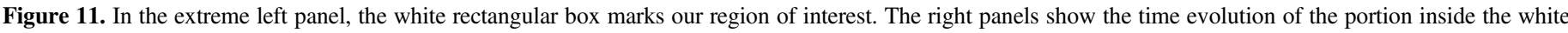

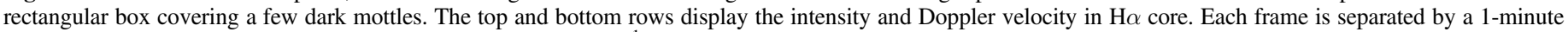
interval. Green contours on the intensity correspond to $-6 \mathrm{~km} \mathrm{~s}^{-1}$ Doppler velocity.

2001; McIntosh et al. 2003; Moretti et al. 2007; Vecchio et al. 2007; Kontogiannis et al. 2010b, 2014). Using Dutch Open Telescope $\mathrm{H} \alpha$ observations with a cadence of $30 \mathrm{~s}$, Kontogiannis et al. (2010a, 2010b) pointed out that there is a strong possibility that power at longer time periods ( $\sim 7$ minutes) may be enhanced as a result of the lifetimes of the mottles. Furthermore, Kontogiannis et al. (2010a) also highlighted that the observed power enhancements, at both photospheric and chromospheric heights, may be closely related to the temporal dynamics of such transients and their lifetimes. In this paper we have explored if transients can influence the power distribution at different heights. The highcadence $(1.34 \mathrm{~s})$ observations presented here allow us to identify and study the dynamics of transient phenomena in greater detail, which was previously not possible due to lower cadence $(\sim 30 \mathrm{~s})$.

The quiet chromosphere is generally dominated by numerous elongated dark structures seen in $\mathrm{H} \alpha$. These include rapidly changing hair-like structures known as mottles and extreme Doppler-shifted events such as RBEs and RREs (for details see the review of Rutten 2012; Tsiropoula et al. 2012). Figure 1 and its associated movie reveal that these structures are associated with the regions of network magnetic fields which appear at the edges of granular cells (Nordlund et al. 2009). It is now generally believed that the dark mottles are the disk counterparts of Type I spicules (Tsiropoula et al. 1994a, 1994b; Tsiropoula \& Schmieder 1997; Christopoulou et al. 2001) and the RBEs are the disk counterparts of Type II spicules (Langangen et al. 2008; De Pontieu et al. 2011; Pereira et al. 2012; Kuridze et al. 2015). The mottles seen in the $\mathrm{H} \alpha$ line have mean velocities of the order of $20-40 \mathrm{~km} \mathrm{~s}^{-1}$ and lifetimes of 3-15 minutes (Tsiropoula et al. 2012). On the other hand, the transients like RBEs and RREs generally exhibit upward motion and rapidly fade away without any signature of downward motion. They have shorter lifetimes (10-120 s), high apparent velocities (50-150 $\mathrm{km} \mathrm{s}^{-1}$ ), and smaller widths (150 and $700 \mathrm{~km}$ ) (Kuridze et al. 2015).

Tziotziou et al. (2003) found that mottles arise at the network boundaries as bursts of material and propagate upward with a velocity around $25 \mathrm{~km} \mathrm{~s}^{-1}$. They also show a tendency to occur several times at the same place with a typical duration of around 5 minutes. Our analysis also indicates that the mottles are jet-like features originating in the network region that propagate upward. Figure 11 shows that the footpoints of mottles display strong blue-ward shift when they originate, but with time they fade away and small redshifts that likely correspond to material falling back along the magnetic-canopy structures are observed. The average blueshift above the network (see Figure 10) indicates that material outflows are present in that region. These outflows are not as strong as they are in individual time frames (see Figure 4), suggesting that outflows are not continuous but rather quasi-periodic in nature. The normalized percentage standard deviation in the photosphere, where 5-minute p-modes dominate, is low (around $2.25 \%$ ) but above the network regions where the RREs and mottles are seen, is quite high (above 10\%, see Figure 7). Higher values of normalized percentage standard deviation cannot be explained solely by the presence of linear MHD waves (observations show that the slow waves generally have an amplitude of less than 5\%; Wang 2011). Numerical models show that the dark mottles observed in $\mathrm{H} \alpha$ are due to material density enhancement (Leenaarts et al. 2006, 2012). So, the fluctuations caused by the rise and fall of material in the form of transients may be responsible for the observed high standard deviation.

Power halos (across all period bands) manifesting in the predominantly photospheric bandpass $(\mathrm{H} \alpha+0.906 \AA$ ) can be explained due to the occurrence of Doppler-shifted transients like RBEs and RREs. The associated movies clearly show the presence of these transients, particularly in the neighborhood of the network field concentrations. Although not strictly periodic, they occur repeatedly (31-15 times in 28 minutes) at the same location and have a lifetime of 10-120 s. Hence, the lifetime and distribution of Doppler-shifted RBEs (see Figure 4 of Sekse et al. 2013) can produce sufficient power enhancement in different periods, as shown from the artificial light curves that corresponds to "case-1" from Section 3.4 as demonstrated in Figure 8.

Imaging data from a passband centered at $0.7 \AA$ from the $\mathrm{H} \alpha$ line core was used to produce the power maps where quiet-Sun "power halos" were positively identified (Kontogiannis et al. 2010a, 2010b, 2014). This is closer to the $\mathrm{H} \alpha$ line core when compared to the predominantly photospheric bandpass at $+0.9 \AA$. Thus, we believe that these previous power-halo detections were more affected than our observations and simulations by Doppler-shifted transients, firmly setting Doppler-shifted transients as the source of the observed halos in the quiet Sun. Note that in our wide-band power maps we do not find significant power enhancement at the regions of halos as observed in $\mathrm{H} \alpha+0.906 \AA$. This confirms the earlier report by Vecchio et al. (2007) who also did not find signatures of power enhancements in the photospheric broadband continuum band (centered at $710 \mathrm{~nm}$ ) close to network regions. There is no reason that power halos should not be observable in wide-band data as they are photospheric. There should be no difference between narrow-band observations at purely photospheric wavelengths and wide-band observations with respect to wave detection. The effective difference we find between the two is the impact of the Doppler-shifted chromospheric transients. 
Similarly, we believe that power from random transients could affect the light curves and influence the power distribution. More generally, the presence of transients can leave a two-dimensional signature visible in power maps obtained in a similar fashion. One such example is "network aureoles," a structure similar to the power halos in the upper photosphere/lower chromosphere reported in Krijger et al. (2001). This effect by transients should be present in the active region power halos as well even though it may be less important in a more stable canopy and stronger wave signal. The power maps are strongly affected close to the network regions where jets occur ubiquitously. In the context of EUV coronal bright points, Samanta et al. (2015) have demonstrated that the quasi-periodic oscillation in transition regions and corona above network regions are due to repeated occurrences of jets around the network regions.

Similarly to the power halos, the magnetic shadow seen closer to the line core $(\mathrm{H} \alpha+0.543$ and $\mathrm{H} \alpha+0.362 \AA)$ in the 3 -minute power band can be affected by the lifetime and distribution of the mottles. It is generally seen that, close to network regions, power above 5 minutes dominates whereas in the internetwork regions, the dominant period is 3 minutes (Dame et al. 1984; Deubner \& Fleck 1990; Bocchialini et al. 1994; Cauzzi et al. 2000; Krijger et al. 2001; Tsiropoula et al. 2009; Gupta et al. 2013; Bostanc1 et al. 2014). The $\mathrm{H} \alpha$ core-intensity signal (see Figure 3 ) is mostly dominated by $\geqslant 5$ minutes oscillations over the entire FOV, whereas the Doppler velocity signal (see Figure 4 ) shows a $\geqslant 5$ minute dominant period very close to the network region and 3 minutes in the internetwork region. Similar behavior was also found by De Pontieu et al. (2007). The reason for this could be that, close to the network center, when the mottles travel upward, we observe blueshifts from material flowing toward the observer, but when these reach the magnetic canopy region, we will not be able to observe any LOS Doppler shifts as the material is flowing horizontally with a quasi-periodicity (the intensity fluctuations can still be observed). Rather, the 3-minute shocks (Carlsson \& Stein 1992, 1997), buffeting the canopy from below in the internetwork region, are observed in the Doppler signal. Hence, the lifetime of mottles will not affect the Doppler power map. The high intensity fluctuations produced by the appearance and disappearance of mottles cause greater power at longer periods, instead of at 3 minutes. We should point out that Kontogiannis et al. (2014) conjectured that the nature of the 7-minute power at the chromospheric heights is not acoustic in nature. Using our simplistic model we tried to mimic the chromospheric power distribution and we find that the suppression of power in the 3-minute period band due to sudden fluctuations (like mottles) is only a few percent (2\%-6\%) whereas the same fluctuations can highly influence longer-period (5-9 minutes) power. Our analysis indicates that the observed long-period oscillation in the $\mathrm{H} \alpha$ core and close to the network in $\mathrm{H} \alpha+0.362$ and $\mathrm{H} \alpha+0.543 \AA$ (see Figure 3) arises due to the longer lifetime of the mottles in the quiet-Sun network regions. From our observations we find that the magnetic shadow regions (network) show 60\%-70\% power reduction compared to the internetwork regions. So, we conclude that although the power can be affected by the lifetime of the mottles, the power suppression due to mottles may not be significant in the 3-minute period. Hence, we conjecture that wave mode conversion may play a key role in forming magnetic shadows in the 3-minute power band. The slow waves may transfer part of their energy upon reaching the canopy layer and convert to fast magnetoacoustic modes. Due to high velocity gradients, the fast mode generally reflects back and forms magnetic shadow (Khomenko \& Collados 2006; Schunker \& Cally 2006). In addition to this process, Rijs et al. (2016) found that fast-to-Alfvén wave mode conversion may play an important role in this process and the the fast wave energy can be converted to transverse Alfvén waves along the field lines. We should also point out that most of the theoretical work on the magnetic portals have not included non-LTE effects, which may play an important role in the coupled chromosphere where radiation effects are also important.

\section{CONCLUSIONS}

We studied the oscillatory behavior of the quiet Sun using $\mathrm{H} \alpha$ observation encompassing network bright points. The power maps at different layers display the well-known "magnetic shadow" and "power halo" features. Previously, these phenomena were interpreted in terms of acoustic waves interacting with inclined magnetic fields. We show that power maps in general can be strongly affected by the lifetimes of these transients. We propose that transients like RBEs, which occur ubiquitously in the solar atmosphere, can have a major effect on the formation of power halos in the quiet Sun. For magnetic shadows around the 3-minute band, the mode conversion seems to be most effective, whereas the power at longer periods is highly influenced by the presence of mottles. We should point out that the shorter time length of the time series will also have some effect on the power analysis. A very long time series should ideally be used for such purposes but high quality ground-based observations are rarely available for prolonged periods. Most of the previous low cadence observations and numerical simulations have ignored the effects of small-scale transients while explaining the magnetic portal. Our high-cadence observations reveal clear presence of these transients and thus waves and transients may simultaneously be present within these structures and can collectively cause the power enhancements and suppression. It will be very difficult to isolate and decouple these effects, although the dominant source for the formation of power halos appears to be the transients from our observation. We hope to quantify the contributions from these two sources in our future work, while studying the phase relation between intensity and velocity at different layers. With high spatial and temporal resolution observations we find that the quiet Sun chromosphere is highly dynamic, where flows, waves, and shocks manifest in the presence of a magnetic field to form an often nonlinear MHD system and future simulations should include all these effects.

We thank the anonymous referee for the valuable comments that enabled us to improve the presentation of this work. This work was supported by UKIERI trilateral research grant of The British Council. The Swedish $1 \mathrm{~m}$ Solar Telescope is operated on the island of La Palma by the Institute for Solar Physics (ISP) of Stockholm University in the Spanish Observatorio del Roque de los Muchachos of the Instituto de Astrofísica de Canarias. This research was supported by the SOLARNET project (www.solarnet-east.eu), funded by the European Commissions FP7 Capacities Program under the Grant Agreement 312495. D.B.J. thanks STFC for an Ernest Rutherford Fellowship in addition to a dedicated standard grant that allowed this project to be undertaken. 


\section{REFERENCES}

Banerjee, D., Erdélyi, R., Oliver, R., \& O’Shea, E. 2007, SoPh, 246, 3

Bocchialini, K., Vial, J.-C., \& Koutchmy, S. 1994, ApJL, 423, L67

Bogdan, T. J., Carlsson, M., Hansteen, V. H., et al. 2003, ApJ, 599, 626

Bostanc1, Z. F., Gültekin, A., \& Al, N. 2014, MNRAS, 443, 1267

Carlsson, M., \& Bogdan, T. J. 2006, RSPTA, 364, 395

Carlsson, M., \& Stein, R. F. 1992, ApJL, 397, L59

Carlsson, M., \& Stein, R. F. 1997, ApJ, 481, 500

Cauzzi, G., Falchi, A., \& Falciani, R. 2000, A\&A, 357, 1093

Centeno, R., Collados, M., \& Trujillo Bueno, J. 2006, ApJ, 640, 1153

Christopoulou, E. B., Georgakilas, A. A., \& Koutchmy, S. 2001, SoPh, 199, 61

Dame, L., Gouttebroze, P., \& Malherbe, J.-M. 1984, A\&A, 130, 331

de la Cruz Rodríguez, J., Löfdahl, M. G., Sütterlin, P., Hillberg, T., \& Rouppe van der Voort, L. 2015, A\&A, 573, A40

De Moortel, I., \& Nakariakov, V. M. 2012, RSPTA, 370, 3193

De Pontieu, B., Erdélyi, R., \& James, S. P. 2004, Natur, 430, 536

De Pontieu, B., Hansteen, V. H., Rouppe van der Voort, L., van Noort, M., \& Carlsson, M. 2007, in ASP Conf. Ser. 368, The Physics of Chromospheric Plasmas, ed. P. Heinzel, I. Dorotovič, \& R. J. Rutten, (San Francisco, CA: ASP), 65

De Pontieu, B., McIntosh, S. W., Carlsson, M., et al. 2011, Sci, 331, 55

Deubner, F.-L., \& Fleck, B. 1990, A\&A, 228, 506

Gupta, G. R., Subramanian, S., Banerjee, D., Madjarska, M. S., \& Doyle, J. G. 2013, SoPh, 282, 67

Heggland, L., Hansteen, V. H., De Pontieu, B., \& Carlsson, M. 2011, ApJ, 743,142

Henriques, V. M. J. 2012, A\&A, 548, A114

Henriques, V. M. J., Kuridze, D., Mathioudakis, M., \& Keenan, F. P. 2016, ApJ, 820, 124

Jefferies, S. M., McIntosh, S. W., Armstrong, J. D., et al. 2006, ApJL, 648, L151

Jess, D. B., Mathioudakis, M., Christian, D. J., Crockett, P. J., \& Keenan, F. P. 2010, ApJL, 719, L134

Jess, D. B., Morton, R. J., Verth, G., et al. 2015, SSRv, 190, 103

Jess, D. B., Reznikova, V. E., Van Doorsselaere, T., Keys, P. H., \& Mackay, D. H. 2013, ApJ, 779, 168

Judge, P. G., Tarbell, T. D., \& Wilhelm, K. 2001, ApJ, 554, 424

Khomenko, E., Centeno, R., Collados, M., \& Trujillo Bueno, J. 2008, ApJL, 676, L85

Khomenko, E., \& Collados, M. 2006, ApJ, 653, 739

Kontogiannis, I., Tsiropoula, G., \& Tziotziou, K. 2010a, A\&A, 510, A41

Kontogiannis, I., Tsiropoula, G., \& Tziotziou, K. 2014, A\&A, 567, A62

Kontogiannis, I., Tsiropoula, G., Tziotziou, K., \& Georgoulis, M. K. 2010b, A\&A, 524, A12

Krijger, J. M., Rutten, R. J., Lites, B. W., et al. 2001, A\&A, 379, 1052

Kuridze, D., Henriques, V., Mathioudakis, M., et al. 2015, ApJ, 802, 26

Langangen, Ø., De Pontieu, B., Carlsson, M., et al. 2008, ApJL, 679, L167
Leenaarts, J., Carlsson, M., \& Rouppe van der Voort, L. 2012, ApJ, 749, 136 Leenaarts, J., Rutten, R. J., Sütterlin, P., Carlsson, M., \& Uitenbroek, H. 2006, A\&A, 449, 1209

Löfdahl, M. G. 2002, Proc. SPIE, 4792, 146

McIntosh, S. W., Fleck, B., \& Judge, P. G. 2003, A\&A, 405, 769

Moretti, P. F., Jefferies, S. M., Armstrong, J. D., \& McIntosh, S. W. 2007, A\&A, 471, 961

Nordlund, A., Stein, R. F., \& Asplund, M. 2009, LRSP, 6, 2

Nutto, C., Steiner, O., \& Roth, M. 2010, AN, 331, 915

Nutto, C., Steiner, O., \& Roth, M. 2012a, A\&A, 542, L30

Nutto, C., Steiner, O., Schaffenberger, W., \& Roth, M. 2012b, A\&A, 538, A79

Pereira, T. M. D., De Pontieu, B., \& Carlsson, M. 2012, ApJ, 759, 18

Pereira, T. M. D., De Pontieu, B., Carlsson, M., et al. 2014, ApJL, 792, L15

Rees, D. E., \& Semel, M. D. 1979, A\&A, 74, 1

Rijs, C., Rajaguru, S. P., Przybylski, D., et al. 2016, ApJ, 817, 45

Roberts, B. 1983, SoPh, 87, 77

Roberts, B. 2000, SoPh, 193, 139

Rosenthal, C. S., Bogdan, T. J., Carlsson, M., et al. 2002, ApJ, 564, 508

Rouppe van der Voort, L., De Pontieu, B., Pereira, T. M. D., Carlsson, M., \& Hansteen, V. 2015, ApJL, 799, L3

Rouppe van der Voort, L., Leenaarts, J., de Pontieu, B., Carlsson, M., \& Vissers, G. 2009, ApJ, 705, 272

Rutten, R. J. 2012, RSPTA, 370, 3129

Samanta, T., Banerjee, D., \& Tian, H. 2015, ApJ, 806, 172

Scharmer, G. B. 2006, A\&A, 447, 1111

Scharmer, G. B., Bjelksjo, K., Korhonen, T. K., Lindberg, B., \& Petterson, B. 2003a, Proc. SPIE, 4853, 341

Scharmer, G. B., Dettori, P. M., Lofdahl, M. G., \& Shand, M. 2003b, Proc. SPIE, 4853, 370

Scharmer, G. B., Narayan, G., Hillberg, T., et al. 2008, ApJL, 689, L69

Schunker, H., \& Cally, P. S. 2006, MNRAS, 372, 551

Sekse, D. H., Rouppe van der Voort, L., \& De Pontieu, B. 2013, ApJ, 764, 164

Shine, R. A., Title, A. M., Tarbell, T. D., et al. 1994, ApJ, 430, 413

Torrence, C., \& Compo, G. P. 1998, BAMS, 79, 61

Tsiropoula, G., Alissandrakis, C. E., \& Schmieder, B. 1994a, A\&A, 290, 285

Tsiropoula, G., \& Schmieder, B. 1997, A\&A, 324, 1183

Tsiropoula, G., Schmieder, B., \& Alissandrakis, C. E. 1994b, SSRv, 70, 65

Tsiropoula, G., Tziotziou, K., Kontogiannis, I., et al. 2012, SSRv, 169, 181

Tsiropoula, G., Tziotziou, K., Schwartz, P., \& Heinzel, P. 2009, A\&A, 493, 217

Tziotziou, K., Tsiropoula, G., \& Mein, P. 2003, A\&A, 402, 361

Uitenbroek, H. 2003, ApJ, 592, 1225

van Noort, M., Rouppe van der Voort, L., \& Löfdahl, M. G. 2005, SoPh, 228, 191

Vecchio, A., Cauzzi, G., Reardon, K. P., Janssen, K., \& Rimmele, T. 2007, A\&A, 461, L1

Wang, T. 2011, SSRv, 158, 397

Zaqarashvili, T. V., \& Erdélyi, R. 2009, SSRv, 149, 355 